\title{
Does the Fluid Elasticity Influence the Dispersion in Packed Beds?
}

\author{
K. Roel Westerterp, Ruud J. Wijngaarden, and Niek B.G. Nijhuis*
}

Reasons are given why the axial dispersion in a gas flowing through a packed bed may be influenced by the elasticity - or compressibility - of the fluid. To support this hypothesis, experiments have been done in a packed column at pressures from 0.13 to $2.0 \mathrm{MPa}$. The elasticity $E$ of a gas is proportional to the pressure $P$ and the compressibility to $1 / P$. The axial dispersion coefficients as determined were found to be a function of the pressure in the packed bed in the turbulent flow region of $3<\mathrm{Re}_{\mathrm{p}}<150$ if the Bodenstein number is plotted as a function of the particle Reynolds number. This is shown to be an artifact. The pressure influence is eliminated, if $\mathrm{Bo}_{\mathrm{m} \text {, ax }}$ is plotted versus the ratio of the kinetic forces over the elastic forces $\varrho u^{2} / E$. Regrettably, $\mathrm{Bo}_{\mathrm{m} \text {, ax }}$ seems to be independent of $\varrho u^{2} / E$. For the moment we only can conclude that $\mathrm{Bo}_{\mathrm{m} \text {, ax }}$ in the turbulent region is a unique function of the velocity of the gas which flows through the packed bed. Although the fact that a constant Bo value is obtained when plotted against $\varrho u^{2} / E$, the experimental results are so intriguing we wanted to make them public already now. The experimental work proceeds.

\section{Introduction}

Axial dispersion data in a fluid flowing through a packed bed are usually given in the form of a Bodenstein number, which in its turn is correlated to the Reynolds number. The Bodenstein number is based on the diameter of the particles in the bed packing and represents the ratio of the mass transport by convection over that by dispersion, being $\mathrm{Bo}=u d_{\mathrm{p}} /\left(\varepsilon D_{\mathrm{ax}}\right)$. In Fig. 1 some literature data on Bo are plotted versus the Reynolds number $u d_{\mathrm{p}} /(\varepsilon v)$ [1]. Here, $u / \varepsilon$ is the interstitial fluid velocity in the packed bed. It can be observed that for gases Bo reaches a value of around 2 for $\mathrm{Re}_{\mathrm{p}}$ values above 10 and remains constant, indicating that a cavity between particles behaves as one mixing cell. For liquids $B o=0.5$ for $0.05<\mathrm{Re}_{\mathrm{p}}<50$ then increases slowly for higher $\mathrm{Re}_{\mathrm{p}}$ values to reach the final value of $\mathrm{Bo}=2$ for $\mathrm{Re}_{\mathrm{p}}$ around 1000. We observe that Hiby [2] already concluded that the resemblance between a packed bed and a cascade of mixers seemed fortuitous.

The axial dispersion of mass in gases and in liquids therefore cannot be described by the Reynolds number alone: besides the ratio of the kinetic energy and viscous dissipation, there must be another phenomenon that governs the dispersion of mass in fluids flowing through a packed bed. The conventional properties of fluids being $\mu, c_{\mathrm{p}}, \varrho, \lambda$, and $D_{\text {mol }}$ do not give us new dimensionless groups which can make correlations of Bo with $\mathrm{Re}_{\mathrm{p}}$ coincide for gases and liquids. The Prandtl number $\operatorname{Pr}=\mu c_{\mathrm{p}} / \lambda$ or the Schmidt number $\mathrm{Sc}=v / D_{\text {mol }}$ fail in this respect, the same as a product like $\mathrm{Re}_{\mathrm{p}} \mathrm{Sc}$. Also the surface tension cannot

\footnotetext{
* Prof. Dr. K. R. Westerterp (author to whom correspondence should be addressed), Chemical Reaction Engineering Laboratories, Chemical Engineering Department, University of Twente, P.O. Box 217, NL-7500 AE Enschede, The Netherlands; Dr. ir. R. J. Wijngaarden, Royal Dutch Shell Laboratories, P. O. Box 38000, NL-1030 BN Amsterdam, The Netherlands; Ir. N.B.G. Nijhuis, Delft University of Technology, P.O. Box 5045, NL-2600 GA Delft, The Netherlands.
}

help us out, because it only plays a role in the case of gas/liquid interfaces, whereas Fig. 1 gives data for only one single fluid flowing through the packing.

In 1990 Westerterp and Wijngaarden [3] developed a hypothesis that the fluid elasticity is the unidentified governing factor; they did so on the basis of experimental work on axial dispersion of heat. In this report this hypothesis is further scrutinised on the basis of axial dispersion of mass.

\subsection{Pellet Scale Hydrodynamics in a Packed Bed}

We will first discuss what happens in a packed bed on a small scale. First, we have to distinguish between flow regimes. At very low rates dispersion phenomena are governed by molecular diffusion. This happens in the following situation: the molecular diffusion time constant $d_{\mathrm{p}}^{2} / D_{\text {mol }}$ is

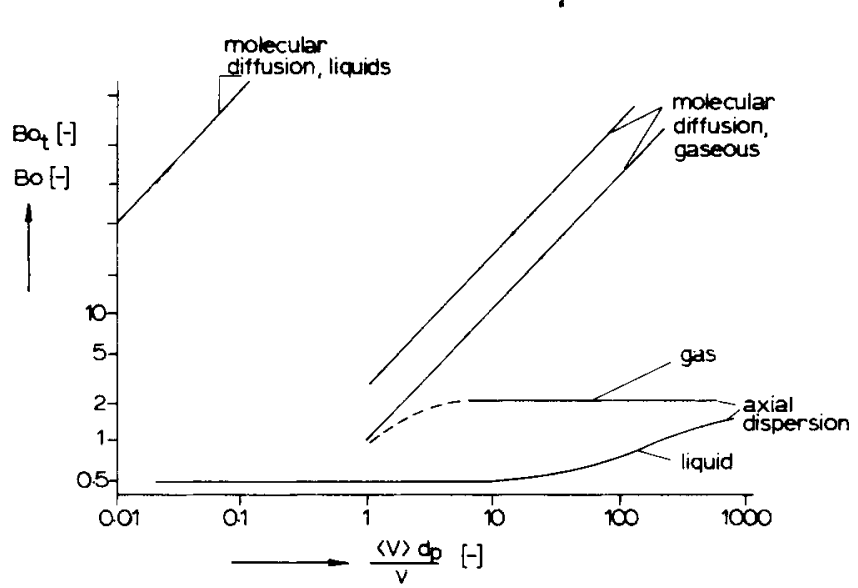

Figure 1. The Bodenstein number for mass dispersion in the axial direction as a function of the Reynolds number $\operatorname{Re}_{\mathrm{p}}$ (based on literature data). 
lower than the residence time $d_{\mathrm{p}} /(u / \varepsilon)$ in a cavity or $u d_{\mathrm{p}} /\left(\varepsilon D_{\mathrm{mol}}\right)=\mathrm{Pe}>1$. This holds for gases for $\mathrm{Re}_{\mathrm{p}}<1$ and for liquids for $\mathrm{Re}_{\mathrm{p}}<0.001$, because $\mathrm{Pe}=\mathrm{Re}_{\mathrm{p}} \mathrm{Sc}$. For values of $\mathrm{Pe}$ much larger than 1 , the molecular diffusion no longer plays a role; the phenomenon of dispersion is fully determined by the hydrodynamics in the packed bed. We are interested only in the regime where $\mathrm{Pe}>1$.

Much has been written on the hydrodynamics in a packed bed but we would like to call the attention to a study published in 1972 by Kirillov et al. (s. Fig. 2). The velocity of a fluid inside a packed bed is distributed in space because the porosity is distributed in space. Kirillov et al. have visualised the flow of gases and liquids in packed beds. Their results indicate that the velocity is also distributed in time. Within the voids between the pellets a flow-through zone exists, in which the fluid flows along tortuous paths in the form of blending and separating streams, and further a non-flow zone in the vicinity of the pellet contact points. A sharp separation boundary is formed between the two zones. This separation boundary is constantly deformed by the existence of rotating and pulsating vortices in the nonflow zone. The pulsation frequency $f$ is directly proportional to the local linear velocity of the streams and inversely proportional to the pellet size so that for gases the Strouhal number $\mathrm{Sr}=\varepsilon f d_{\mathrm{p}} / u \approx 0.5$ for $20<\mathrm{Re}_{\mathrm{p}}$. For liquids, $\mathrm{Sr} \approx 0.5$ only holds for $\mathrm{Re}_{\mathrm{p}}>800$. For packed beds as they are used in chemical industry, this means that the jets erupting from the voids between the pellets are pulsating and vibrating with frequencies from 10 to $100 \mathrm{~Hz}$. Further, Kirillov et al. [4] observed that the toroidal vortices occur in the wake of the particles with respect to the main fluid stream and not in the neighbourhood of the contact spots lying in the main stream direction itself.

The local velocity has been studied extensively in the literature, e.g., Drahos et al. [5] measured the velocity vector components in the axial, radial, and angular directions, $v_{z}, v_{\mathrm{r}}$, and $v_{\theta}$. An example of their results is presented in Fig. 3, where the reduced velocities, being the actually measured velocity $v_{z}, v_{\mathrm{r}}$, and $v_{\theta}$ divided by the interstitial velocity $u / \varepsilon$ in the bed are plotted versus the distance along

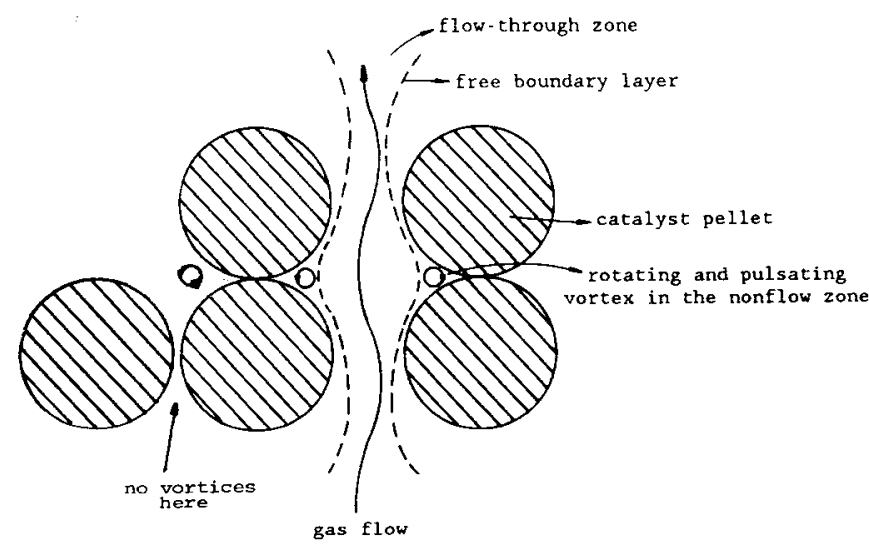

Figure 2. Flow-through and non-flow zones in a packed bed. a circumference. Notice the tremendous amount of scatter in all velocity components: this is consistent with the picture, Kirillov et al. [4] sketched of a random packed bed with its blending and separating streams and pulsating vortices. Also we see that the radial and angular components are considerable when compared to the axial component.

Other authors measured the norm of the vector $v$ only. An example of this is given in Fig. 4 taken from Price [6]. Also in $v$, there is a large amount of scatter (see also Vortmeyer and Schuster [7]). The experiments of Kirillov et al. [4] show that it is impossible to describe the behaviour of the fluid flow through the packing using only the Reynolds number. The hydrodynamics of the fluid flow are much more complicated.

\subsection{Description of Dispersion Phenomena}

Other properties of the packed bed with a fluid flowing through are strongly related to the hydrodynamics; an example is radial dispersion of mass and heat, where radial dispersion of heat rather than of mass is usually important, e.g., for cooled tubular reactors. Usually, this radial disper-

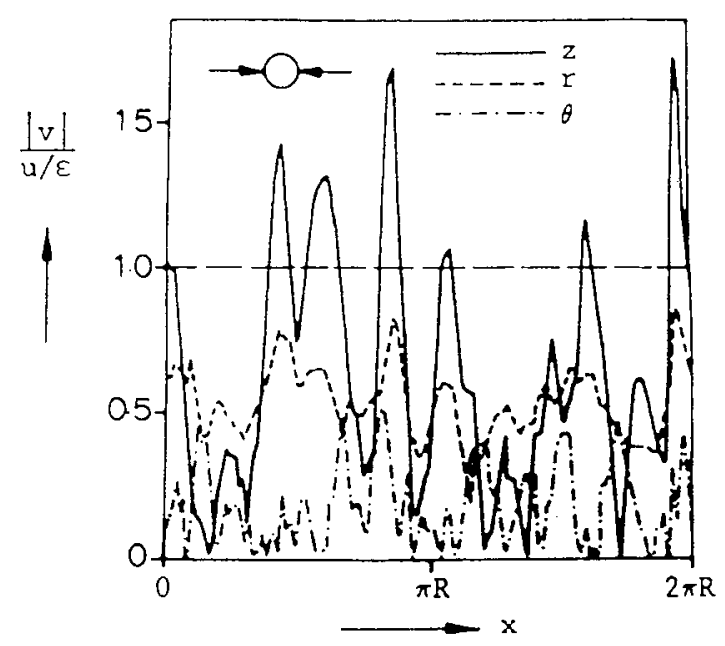

Figure 3. Axial, radial, and angular velocity components versus the distance along a circumference. Particle size as indicated. Results from Drahos et al. [5].

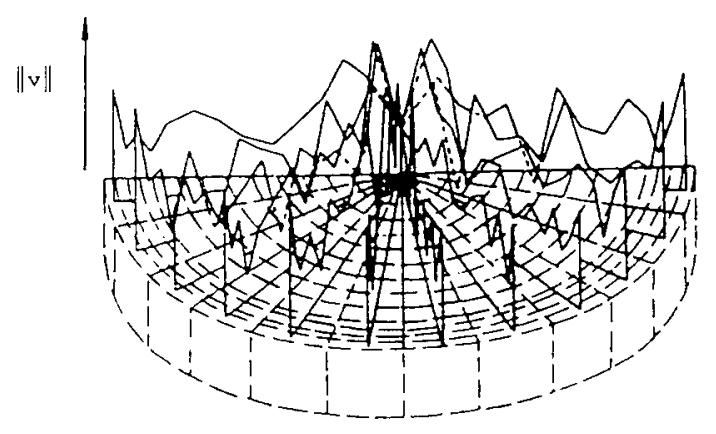

Figure 4. Norm of the velocity vector as a function of the location on the bed exit plane. Only half the bed is shown, from Price [6]. 
sion of heat in packed beds is described with lumped parameters as the effective radial heat conductivity $\lambda_{\text {eff,r }}$ and the heat transfer coefficient $\alpha_{w}$ at the wall of the packed bed.

To describe axial dispersion of mass in packed beds, in principle it is assumed that a sort of random walk is superimposed on the velocity front of the fluid. The non-flow and flow zones near the pellet vicinities can be regarded as the source of this random walk. A molecule will remain in one of the zones for a certain period of time, having a certain chance to remain in its zone or to switch over to the other zone. Hence, there is a certain axial velocity difference between different molecules, which leads to axial dispersion. The residence time in both zones is the order of the contact time between the pellet and the fluid. By calculating for successive time steps how the molecules are distributed over the different zones of the packed bed, the residence time distribution of the molecules can be estimated. Molecules in the non-flow and flow zones are in different states. We can assume as many states as we want: e.g., the molecule going back, standing still, going forward with twice or half the superficial velocity, being absorbed on the pellet, etc.

To summarise, a number of models have been developed to describe bed scale behaviour. This has only been successful in a few simple cases. For industrial applications, we still have to resort to deterministic bed scale models which use lumped parameters, e.g., the effective axial and radial heat conductivities, the heat transfer coefficient between the wall and the packed bed, and the axial and radial mass dispersion coefficients. Those lumped parameters always find their origin in pellet scale behaviour. Because of the complexity of the deterministic bed scale models it is very hard, if not impossible, to link the lumped parameters to the physical phenomena occurring on a pellet scale. Therefore, only empirical correlations are given for the lumped parameters, whereas the physicochemical understanding of the correlations and their constants is still lacking.

\subsection{What is the Second Dimensionless Group to Describe Dispersion?}

Westerterp and Wijngaarden [3] report on their experimental work of axial and radial heat transfer at different pressures. They correlated the results by: ${ }^{1)}$

$$
\mathrm{Bo}_{\mathrm{h}, \mathrm{r}}=\frac{7.9}{1+235 / \mathrm{Re}_{\mathrm{p}}}
$$

and

$$
\mathrm{Bo}_{\mathrm{h}, \mathrm{ax}}=\frac{0.65}{1+65 P / \mathrm{Re}_{\mathrm{p}}}
$$

For very high gas velocities, $\mathrm{Bo}_{\mathrm{h}, \mathrm{r}}$ approaches 7.9 and $\mathrm{Bo}_{\mathrm{h}, \mathrm{ax}}$ approaches the value of 0.65 . This means that under

1) List of symbols at the end of the paper. all conditions the dispersion of heat in the axial direction is much higher than in the radial direction. This anisotropy can be understood in view of the fact that the toroidal vortices only exist in the wake of particles in the direction of the main flow and not perpendicular to it. So heat transport will be much easier in the axial than in the radial direction.

The term $P / \operatorname{Re}$ should be representative for a second dimensionless number, which is related to the hydrodynamics and specifically in the axial direction. So the second dimensionless group in fact contains $u^{-1}$, because the pressure effect is cancelled. It may also contain $\lambda, v, c_{\mathrm{p}}, \varrho, g$, and $d_{\mathrm{p}}$, which were not varied during their experiments.

As to the physical properties which might influence heat transfer via the hydrodynamics, we must consider the acceleration due to gravity. However, it is well known that the gravitational field only influences the hydrodynamics in packed beds when velocities are low and free convection becomes significant. This probably can be ruled out for our experiments. The Prandtl number, which cannot be related to the hydrodynamics, can also be ruled out.

From the experiments of Kirillov et al. [4] as described earlier, Westerterp and Wijngaarden found that the particle Strouhal number $\operatorname{Sr}=\varepsilon f d_{\mathrm{p}} / u$, in which $f$ is the pulsation and rotation frequency of the vortices in the non-flow zone, is significant for flow inside packed beds. Due to the pulsations and rotations the vortices are continuously expanded and recompressed. Moreover, as seen for example in Fig. 3, the gas streams in the flow-through zone continuously collide with the particles, are deflected, change direction, etc. Westerterp and Wijngaarden therefore suggested that the elasticity $E$ of the flowing medium as given by

$E=-V\left[\frac{\partial P}{\partial V}\right]$

could be significant for hydrodynamics inside the packed bed. It could play a role in the process of the continuing deformation of the vortices in the non-flow zone by the streams in the flow zone and vice versa. For ideal gases, $E$ equals the pressure $P$. As the elasticity is the reciprocal of the compressibility, it is arbitrary which of the two parameters is taken to relate to the pulsations and rotations in the vortices.

The pressure can be introduced in a dimensionless group as follows:

$\sqrt{\frac{P}{\varrho u^{2}}} \approx \sqrt{\frac{E}{\varrho u^{2}}}=\frac{1}{\sqrt{\mathrm{Ho}}}$

which represents the square root of the ratio of the elastic forces over the inertia forces. It is also equal to $1 / \sqrt{\mathrm{Ho}}$, where Ho is the Hooke number. For gases at atmospheric pressure $E_{\mathrm{G}}=10^{5} \mathrm{~N} / \mathrm{m}^{2}$, and for water $E_{\mathrm{L}}=2.18 \cdot 10^{9} \mathrm{~N} /$ $\mathrm{m}^{2}$.

We now can rewrite the relation we have found for axial dispersion of heat, realising that $\mathrm{Bo}_{\mathrm{h}, \mathrm{ax}}=\left(\mathrm{Re}_{\mathrm{p}} \operatorname{Pr}\right) / \Gamma_{\mathrm{ax}}$, 
where $\Gamma_{\mathrm{ax}}=\lambda_{\text {eff }} / \lambda$. The following correlation is then obtained for the experiments [3].

$\Gamma_{\text {ax }}=\frac{\operatorname{Re}_{\mathrm{p}} \operatorname{Pr}}{A}\left(1+\frac{B}{\sqrt{\mathrm{Ho}}}\right)$

or for one single gas $\Gamma_{\mathrm{ax}}=C \mathrm{Re}_{\mathrm{p}}+D P d_{\mathrm{p}}$, where $A, B, C$ and $D$ are constants. We can observe that $\Gamma_{\text {ax }}$ is proportional to $\operatorname{Re}_{\mathrm{p}}$ and further in a second term proportional to the pressure $P$ of the system and also to $d_{\mathrm{p}}$, which is confirmed by the work of Vortruba et al. [8].

We now want to investigate whether also for axial dispersion of mass - the experiments discussed above refer to dispersion of heat - there is an influence of pressure on the dispersion coefficient. If there is a similarity between dispersion of heat and of mass - which, if we neglect heat conductions through particles, is to be expected, because both are caused by the same flow mechanisms - we further may expect a correlation of the same shape as for heat dispersion, now being:

$\frac{1}{\mathrm{Bo}_{\mathrm{m}, \mathrm{ax}}}=\frac{1}{A^{\prime}}\left(1+B^{\prime} \frac{P}{\operatorname{Re}_{\mathrm{p}}}\right)$

If only one system of gases is used, we expect to find a correlation like

$D_{\mathrm{ax}} / D_{\mathrm{mol}}=\Gamma_{\mathrm{ax}, \mathrm{m}}=A^{\prime \prime} P u+B^{\prime \prime} P d_{\mathrm{p}}$

Below results of experiments are discussed, which were set up to verify the validity of the shape of the latter correlation.

\section{Experimental Set-up}

\subsection{Installation}

The experimental installation, shown in Fig. 5, consists of a packed column with an internal diameter $d_{\mathrm{t}}$ of $0.05 \mathrm{~m}$ and a length $L$ of $3.73 \mathrm{~m}$. The column consists of an inlet section of $0.70 \mathrm{~m}$ and a measurement section of $3.025 \mathrm{~m}$. The column is packed with glass spheres with a diameter of $d_{\mathrm{p}}=2.21 \pm 0.29 \mathrm{~mm}$. The bed porosity is 0.36 and the number of packing layers equals \pm 1370 . The pressure in the column can be varied from 0 to 20 bar.

The gas flow consists of nitrogen of $99.5 \%$ purity, in which a small pulse of helium is injected as tracer gas. The nitrogen flow can be controlled from 0 to $5 \mathrm{~N} \mathrm{~m}^{3} / \mathrm{h}$. To this end two mass flow controllers have been installed. The tracer gas helium is injected into the inlet piping by a sixway valve as shown in Fig. 6 . Under stationary conditions nitrogen flows through this valve, to which a small loop of pipe with a volume of $3.14 \mathrm{ml}$ is connected. This loop can be filled with helium. The amount of injected helium can be adjusted by varying the helium pressure in the loop from 0 to 25 bar. At the moment of injection, the valve is turned $60^{\circ}$ and nitrogen purges the injection loop and the helium flows into the column. The injection peak cannot be described by a $\delta$-dirac function, as a consequence the concentration of the helium has to be measured at two locations in the packed column to obtain dispersion data.

At the inlet and outlet of the measurement section a small continuous flow sample of 0 to $1.5 \mathrm{l}_{\mathrm{N}} / \mathrm{h}$ is taken. At the first sampling point - which is situated at the bottom of the tube, just above the inlet section - a sampling device has been constructed, which takes in gas over the whole cross section. At the second sampling point, which is situated just

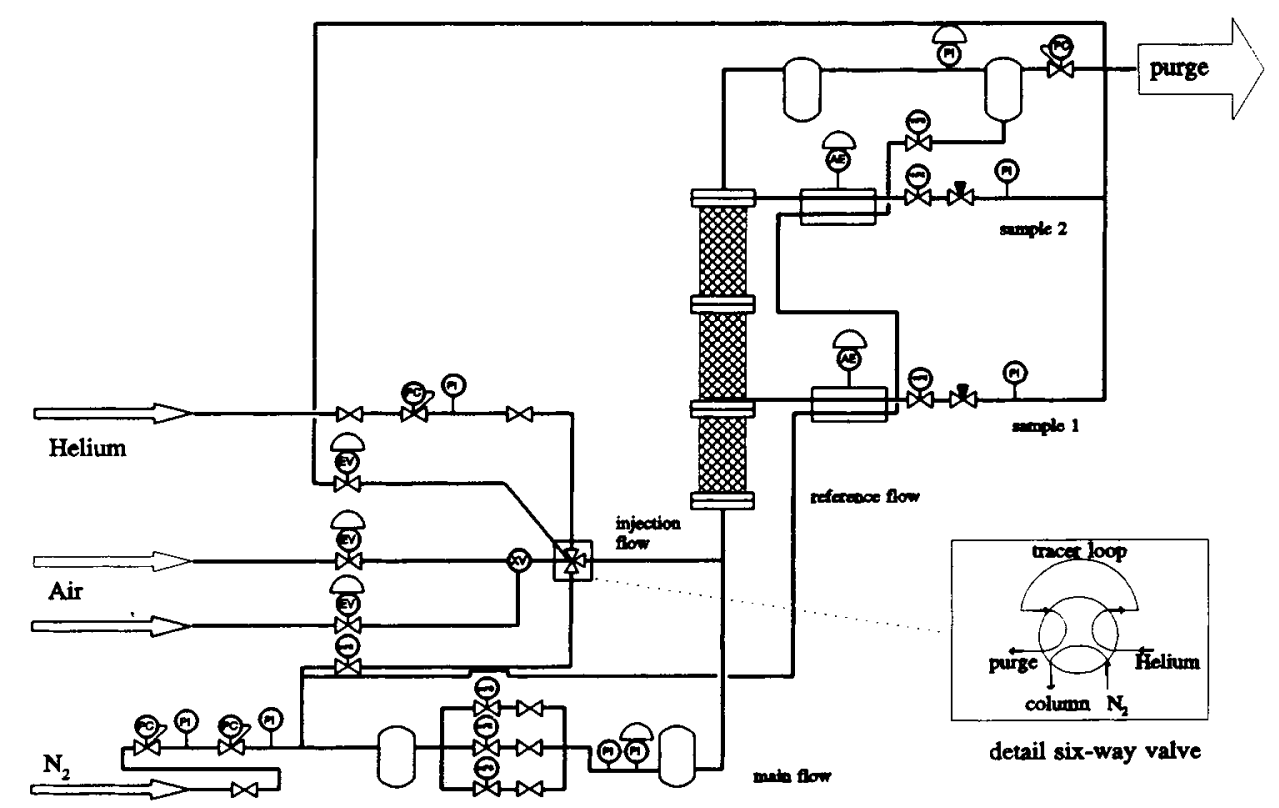

Figure 5. The experimental installation. 


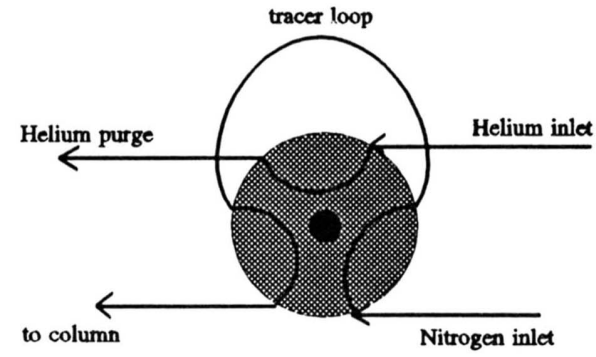

Injection valve position during injection

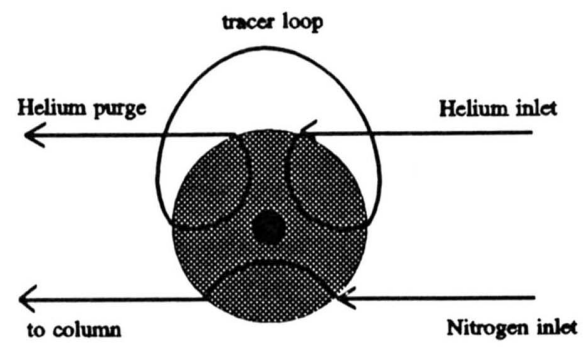

Injection valve position during normal operation

Figure 6. The injection system.

below the top of the tube, some gas is taken off via a small sampling hole in the wall of the tube. These sampling points are shown in Fig. 7. The helium concentration is measured by means of a thermal conductivity detection system (TCD). The $\mathrm{mV}$ signal is a linear function of the tracer concentration.

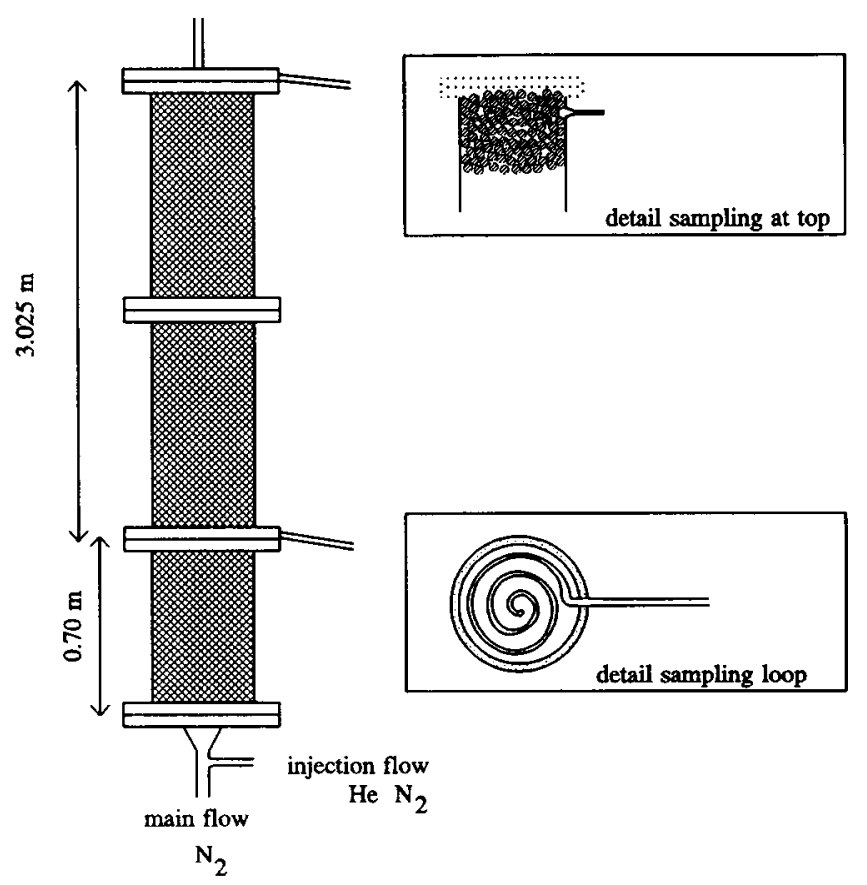

Figure 7. The location and shape of the sampling points.

\subsection{Execution of an Experiment}

At the start of an experiment, the six-way valve is turned to inject tracer material. At this moment time is set to zero. During the time interval in which the helium peak passes the first sampling point, the concentration of the helium is measured as a function of time.

Then concentration measurement is stopped and just before the helium is expected to enter the second sampling point, the measurement as a function of time is resumed via the second sample point.

A high measurement resolution is important for a correct determination of the peaks. Therefore, 1200 data points for time and katharometer signals are taken, so 600 data points for each peak. It is important to measure concentration also a short time just before and after the signal passes.

This information is necessary to define the base line of the signal, when the sample does not contain tracer material. Usually the peak itself consists of 300 to 400 points and the baseline of 200 to 300 data points. The maximum data acquisition rate was $140 \mathrm{~Hz}$.

The output of an experiment is a data file, which contains the estimated Reynolds number of the measurement, the absolute pressures at bottom and top of the column and the 1200 measurement points of the time and the value of the TCD signal.

\subsection{Evaluation of an Experiment}

From the data of each experiment the Bodenstein number $\mathrm{Bo}_{\mathrm{m}, \mathrm{ax}}$ and the average residence time $\tau$ of the model for plug flow with axial dispersion [1] have to be determined.

The raw data for one experiment are shown in Fig. 8. Their conversion into residence time distribution curves takes

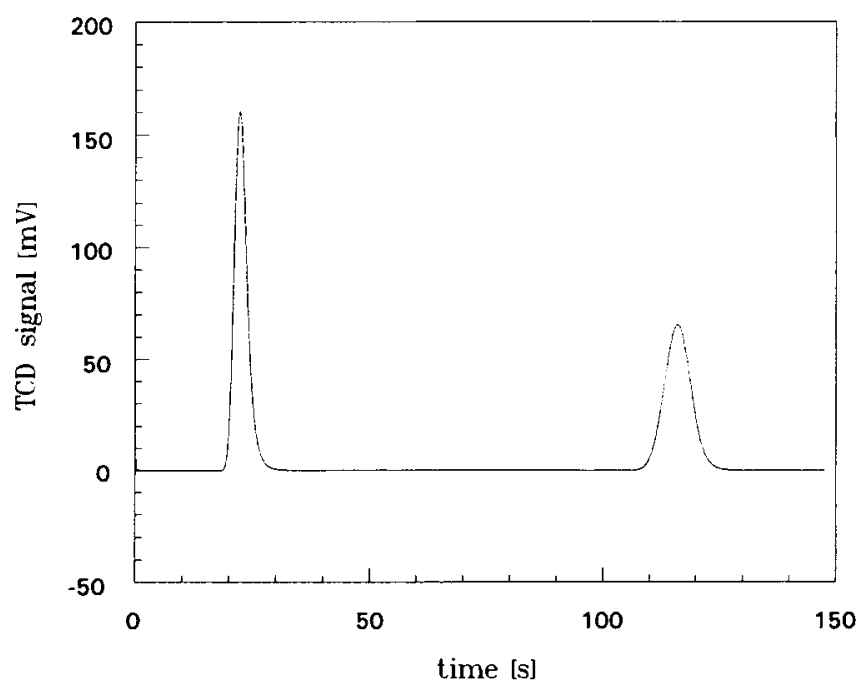

Figure 8. The signal as measured at the two sampling points. 
four steps. The first step is the filtering of the raw data by the method of Golay and Savitzky [9], using a third order polynomial equation. After filtering, the baseline is reduced to zero. This is done by using linear regression via the least squares method on the data points, not belonging to the peak. The start or the end of a peak is determined by comparing the difference of two subsequent data points to a chosen value starting at the center of the peak and going to the left or to the right, respectively. As long as the difference between two points is larger than the chosen value, the peak has not ended. The result is shown in Fig. 9. Next step is cutting off the signals at beginning and end: We do so when the TCD signal is smaller than $1 \%$ of the maximum value of the peak. The fourth and last step is the normalisation of the peaks. To convert the measured peaks into residence time distribution curves, the surface of a signal must be set to unity. By numerical integration using the Simpson rule the surface is determined. Then the signals are normalised by division by the total surface (s. Fig. 10).

The raw data are converted into the residence time distribution curves $E(t)$. The parameters $\mathrm{Bo}_{\mathrm{m}, \mathrm{ax}} L / d_{\mathrm{p}}$ and $\tau$ now can be evaluated, e.g., using the weighted moments method with a constant value of the Laplace variable $s$, according to Michelsen and Østergaard [10].

The criterion used for the best values of the parameters $\mathrm{Pe}$ and $\tau$ is according to Westerterp and Van Gelder [11] that

$\Delta A=\int_{0}^{\infty}\left|C_{\text {output }}^{\text {experimental }}(t)-C_{\text {output }}^{\text {theoretical }}(t)\right| \mathrm{d} t$

must be minimal.

For each experiment the parameters $P e$ and $\tau$ were calculated for values of $s \tau$ between 0 and 50 , the theoretical response curves were calculated by convoluting the measured and normalised input and the transfer function for the axial dispersion model with open-open boundary conditions. Then the differences $\Delta A$ were calculated and the best fit parameters were found at the minimum value of $\Delta A$. An example of a fit is shown in Fig. 11, where the experimental and theoretical response curves can be compared.

\section{Correlation of Results}

Measurements of the residence time distribution have been carried out at pressures of $0.13,0.5,1.0,1.5$, and $2.0 \mathrm{MPa}$, respectively, and at interstitial Reynolds numbers between 3 and 300. Some results are shown in Figs. 12 and 13 at column pressures of 0.13 and $1.5 \mathrm{MPa}$, respectively. In Fig. 14 all series at the five different pressures are presented. We can observe a noticeable influence of pressure if $B o$ is plotted versus $\mathrm{Re}_{\mathrm{p}}$. In Fig. 15 we have plotted all results at all pressure levels as $\mathrm{Bo}^{-1.0}$ versus $\mathrm{Ho}^{-0.5}$ and have drawn the best fitting straight line through the data points for Ho $>10^{-8}$. The correlation of the straight line in Fig. 15 is $\frac{1}{\mathrm{Bo}_{\mathrm{m}, \mathrm{ax}}}=0.40+6 \cdot 10^{-6} \cdot \mathrm{Ho}^{-0.5}$

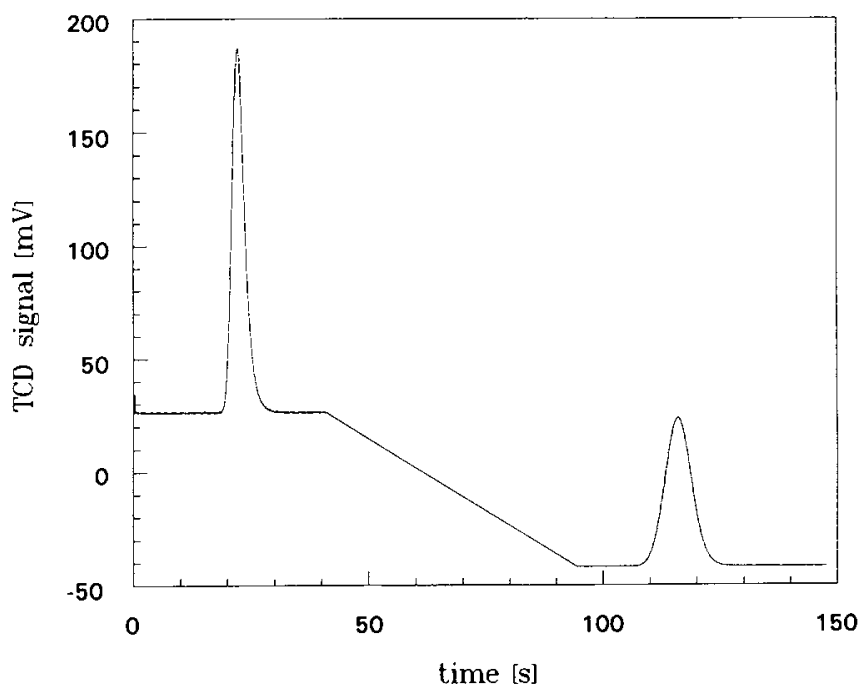

Figure 9. The corrected signals at inlet and outlet of the test section.

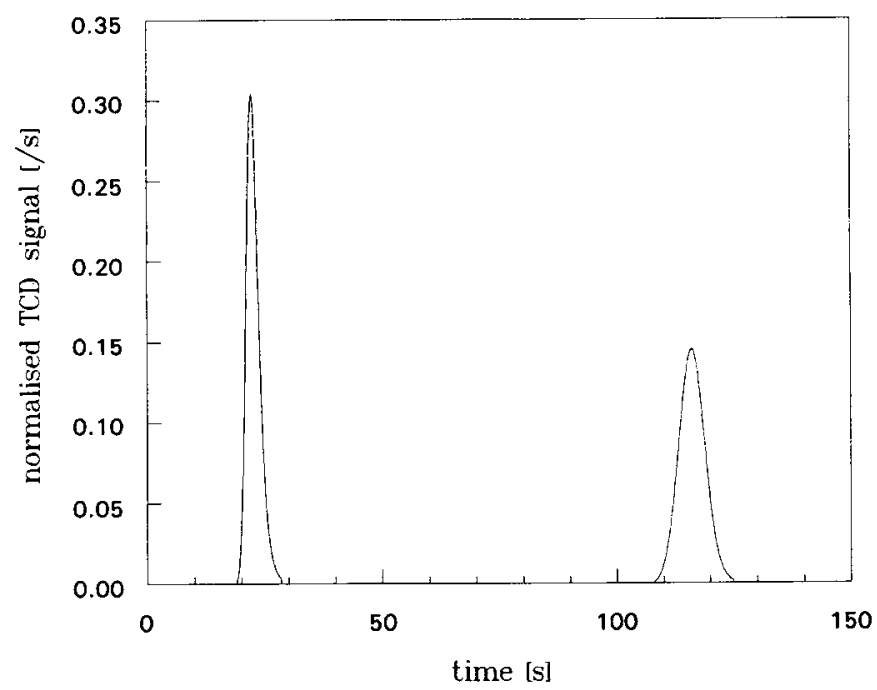

Figure 10. The normalised signals.

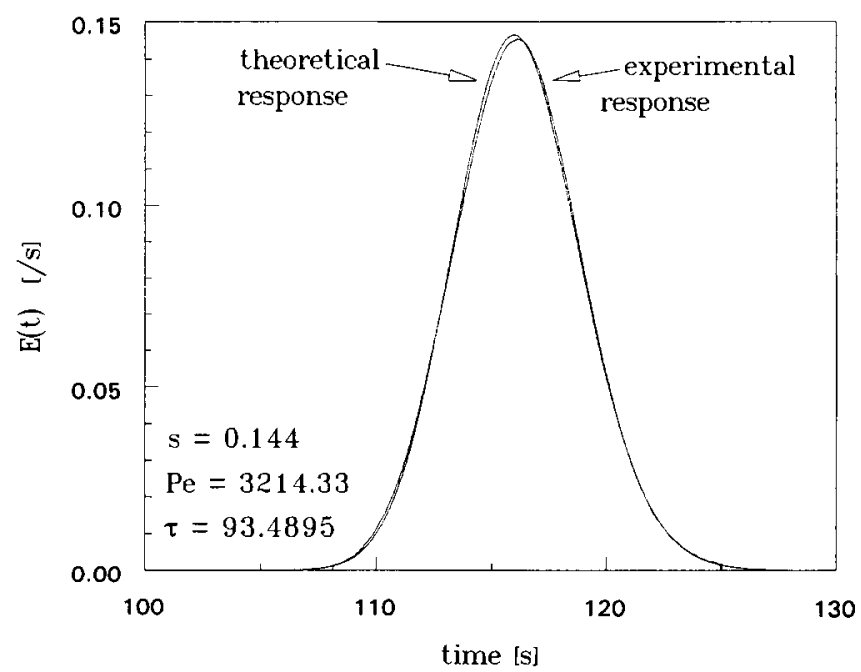

Figure 11. An example of a best fitted theoretical response and the experimental response. 


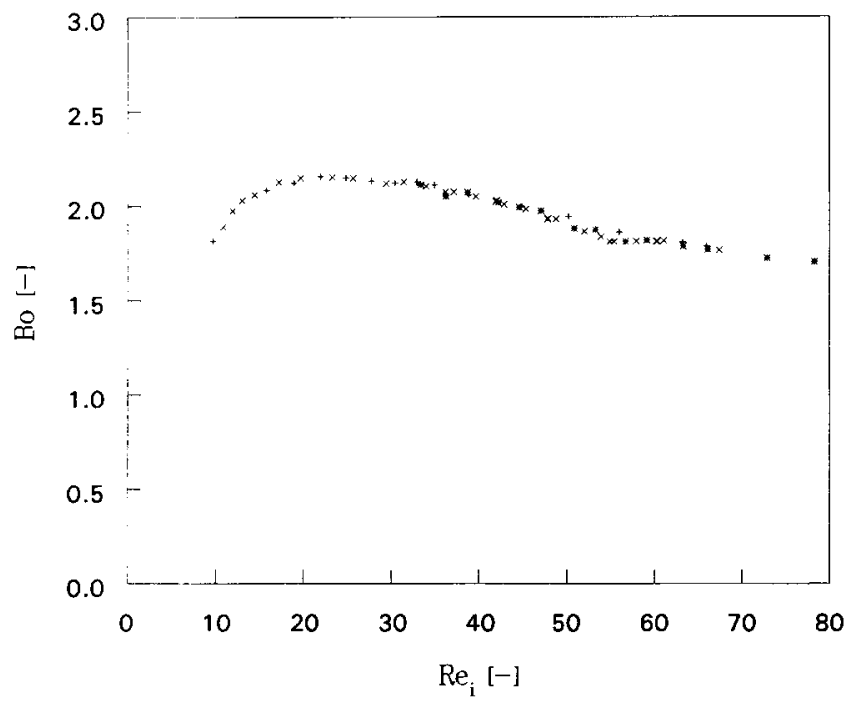

Figure 12. The experimental Bodenstein numbers at $0.13 \mathrm{MPa}$.

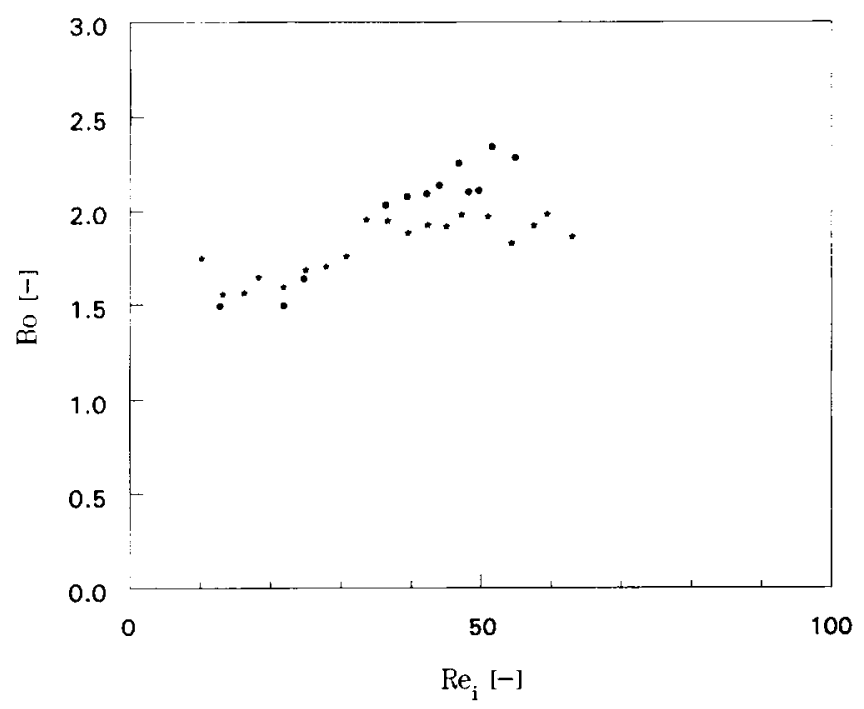

Figure 13. The experimental Bodenstein numbers at 1.5 MPa.

This relation can be rewritten as:

$\mathrm{Bo}_{\mathrm{m}, \mathrm{ax}}=\frac{2.5}{1+\frac{15 \cdot 10^{-6}}{\sqrt{\mathrm{Ho}}}}=\frac{166.7 \cdot 10^{3} \sqrt{\mathrm{Ho}}}{1+66.7 \cdot 10^{3} \sqrt{\mathrm{Ho}}}=\frac{568 u}{1+227 u}$

which is valid for $u>30 \cdot 10^{-3} \mathrm{~m} / \mathrm{s}$, that is in the region where the gas flows turbulently through the bed.

\section{Discussion and Conclusions}

The relation suggests that for large values of Ho of say above $25 \cdot 10^{-9}$, or at large linear gas flow velocities above $0.25 \mathrm{~m} / \mathrm{s}$ through the bed, the value of Bo approaches 2.5 .

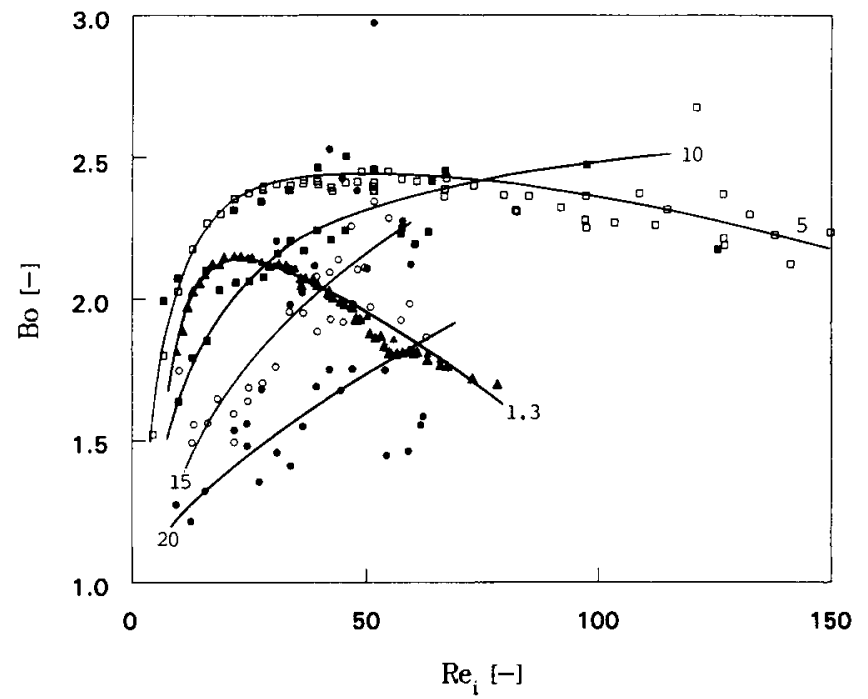

Figure 14. All results for Bodenstein numbers at the absolute column pressures of $0.13,0.5,1.0,1.5$, and $2.0 \mathrm{MPa}$, respectively. For symbols see Fig. 15.

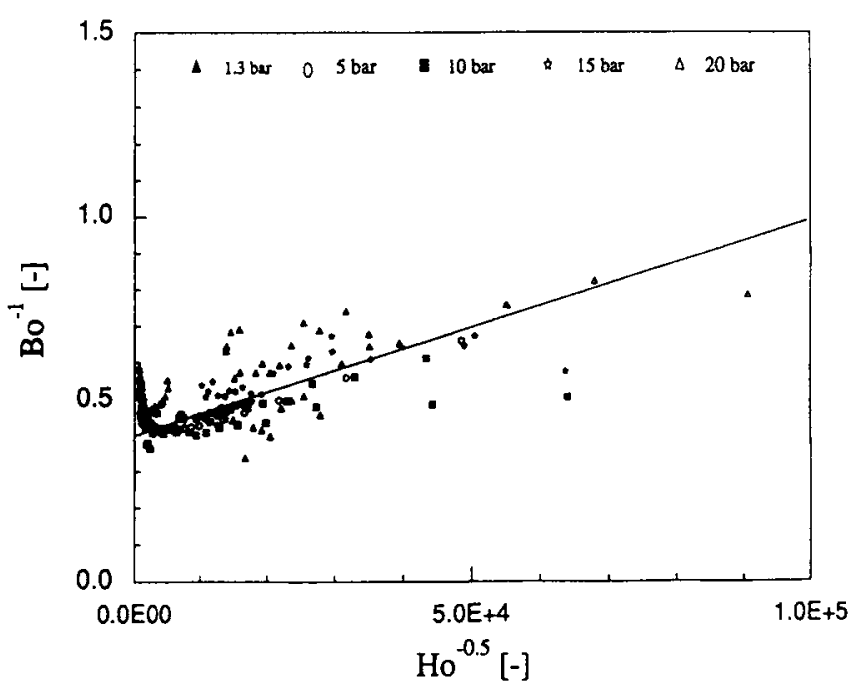

Figure 15. All results, now plotted as $1 /$ Bo versus $\sqrt{\varrho u^{2} / P}$. Parameter is column pressure. Data for $\operatorname{Re}_{p}>12$ and $u>0.03 \mathrm{~m} / \mathrm{s}$.

We might have expected a value of $\mathrm{Bo}=\mathbf{2 . 0}$ for the reasons explained in the introduction. The deviation, however, is within the accuracy of our experimental results. We further can state that in the range of $3<\operatorname{Re}_{\mathrm{p}}<150$ for gases flowing through a packed bed the Bodenstein numbers found in this turbulent flow region can not be represented as a function of $\mathrm{Re}_{\mathrm{p}}$ at different column pressures; it is dependent on it. If correlated as $\mathrm{Bo}_{\mathrm{m} \text {, ax }}$ as a function of a Ho number, a line is obtained, where all points at different pressures do coincide and a unique function of the flow velocity of the gas is obtained. In the latter relation the accuracy is not high (s. Fig. 15), nevertheless, an effect of the pressure cannot be distinguished anymore.

In the last Equation and also in Fig. 15, we observe that for lower velocities $\mathrm{Bo}_{\mathrm{m} \text {, ax }}$ only depends on $u$ in the region of 
turbulent flow, say for $30 \cdot 10^{-3}<u<150 \cdot 10^{-3} \mathrm{~m} / \mathrm{s}$. For values of $1 / \sqrt{\mathrm{Ho}}<2000$ or at superficial velocities of above $0.15 \mathrm{~m} / \mathrm{s}$ or $\mathrm{Re}_{\mathrm{p}}>60$, we observe a decrease of $\mathrm{Bo}_{\mathrm{m} \text {, ax }}$. This implicates that for higher gas velocities $D_{\mathrm{ax}}$ increases with increasing gas velocity through the packed bed. This will be further investigated.

The question might arise whether a correlation of $\mathrm{Bo}_{\mathrm{m}, \mathrm{ax}}$ versus $P e=R_{p} S c$ could explain the observed influence of pressure in plots of $\mathrm{Bo}_{\mathrm{m}}$, ax versus $\mathrm{Re}_{\mathrm{p}}$. This cannot be the case, because $\mathrm{Sc}=v / D_{\text {mol }}$ is independent of pressure. In practice the viscosity $\mu$ is independent of the density $\varrho$, proportional to the molecular diffusion coefficient $D_{\mathrm{mol}}$, and reciprocally proportional to the pressure.

In our experiments we have checked the pressure drop over the test section. The pressure drop always was negligible compared to the absolute pressure in the column. So the phenomenon we observed cannot be a disguised pressure drop effect.

We have given reasons, why in the turbulent region the axial dispersion coefficient in gases flowing through packed beds is not a function of the pressure, and have shown that a previous speculation that Bo can be correlated with a Hooke number also has not been proven. Up to now we have found $\mathrm{Bo}_{\mathrm{m}, \mathrm{ax}}$ is only a unique function of the flow velocity of the gas flowing through the packed bed and not of the ratio of the elastic forces over the inertia forces. We will extend the range of our experiments to lower Reynolds numbers and to particles of different diameters. Further, we also will measure axial dispersion coefficients in liquids with different compressibilities. To this end, we have to modify our experimental installation. However, we found our first results so intriguing that we want to make them public already now.

\section{Acknowledgement}

We owe very much to Ir. I. Smit and Ir. M. S. J. Kamphuis, who constructed the experimental installation and performed the preliminary tests and experiments.

Received: June 19, 1995 [CET 755]

\section{Symbols used}

$\begin{array}{ll}c_{\mathrm{p}} & [\mathrm{J} / \mathrm{kg} \mathrm{K})] \\ D_{\mathrm{mol}} & {\left[\mathrm{m}^{2} / \mathrm{s}\right]} \\ D_{\mathrm{ax}} & {\left[\mathrm{m}^{2} / \mathrm{s}\right]} \\ E & {\left[\mathrm{~N} / \mathrm{m}^{2}\right]} \\ d_{\mathrm{p}} & {[\mathrm{m}]} \\ f & {[1 / \mathrm{s}]} \\ P & {\left[\mathrm{~N} / \mathrm{m}^{2}\right]} \\ u & {[\mathrm{~m} / \mathrm{s}]} \\ v & {[\mathrm{~m} / \mathrm{s}]}\end{array}$

specific heat molecular diffusivity mass dispersion elasticity particle diameter pulsation frequency system pressure fluid velocity based on empty cross section (superficial fluid velocity) local velocity

\begin{tabular}{|c|c|c|}
\hline$\alpha$ & {$\left[\mathrm{J} /\left(\mathrm{m}^{2} \mathrm{~s} \mathrm{~K}\right]\right.$} & heat transfer coefficient \\
\hline$\Gamma$ & {$[-]$} & $\begin{array}{l}\text { dimensionless effective thermal conductivity } \\
\text { or } D_{\mathrm{ax}} / D_{\mathrm{mol}} \text { for mass dispersion }=\lambda_{\mathrm{eff}} / \lambda\end{array}$ \\
\hline$\varepsilon$ & {$[-]$} & bed porosity \\
\hline$v$ & {$\left[\mathrm{~m}^{2} / \mathrm{s}\right]$} & kinematic viscosity $=\mu / \varrho$ \\
\hline$\lambda$ & {$[\mathrm{J} /(\mathrm{m} \mathrm{s} \mathrm{K})]$} & heat conductivity \\
\hline$\lambda_{\text {eff }}$ & {$[\mathrm{J} /(\mathrm{m} \mathrm{s} \mathrm{K})]$} & $\begin{array}{l}\text { effective thermal conductivity of the bed in } \\
\text { the axial direction }\end{array}$ \\
\hline$\mu$ & {$\left[\mathrm{N} /\left(\mathrm{m}^{2} \mathrm{~s}\right)\right]$} & dynamic viscosity \\
\hline$\varrho$ & {$\left[\mathrm{kg} / \mathrm{m}^{3}\right]$} & density \\
\hline
\end{tabular}

\section{Dimensionless numbers}

$\begin{array}{ll}\mathrm{Bo}_{\mathrm{m}, \mathrm{ax}} & \begin{array}{l}\text { Bodenstein number for mass disper- } \\ \text { sion }=u d_{\mathrm{p}} / \varepsilon D_{\mathrm{ax}}\end{array} \\ \mathrm{Bo}_{\mathrm{h}} & \begin{array}{l}\text { Bodenstein number for heat dispersion } \\ \varrho=c_{\mathrm{p}} u d_{\mathrm{p}} /\left(\varepsilon \lambda_{\mathrm{eff}}\right)\end{array} \\ \mathrm{Ho} & \text { Hooke number }=\varrho u^{2} / E \\ \mathrm{Pe} & \text { Peclet number for molecular dispersion of } \\ & \text { mass }=u d_{\mathrm{p}} /(\varepsilon D) \\ \mathrm{Pr} & \text { Prandtl number }=c_{\mathrm{p}} \mu / \lambda \\ \mathrm{Re} & \text { Reynolds number }=u d_{\mathrm{p}} /(\varepsilon v) \\ \mathrm{Sc} & \text { Schmidt number }=v / D \\ \mathrm{Sr} & \text { Strouhal number }=\varepsilon f d_{\mathrm{p}} / u\end{array}$

\section{Indices}

$\begin{array}{ll}\text { ax } & \text { in axial direction } \\ \text { eff } & \text { effective } \\ \mathrm{G} & \text { of the gas } \\ \mathrm{h} & \text { for heat } \\ \mathrm{L} & \text { of the liquid } \\ \mathrm{m} & \text { for mass } \\ \mathrm{r} & \text { in the radial direction } \\ \mathrm{z} & \text { in the axial direction } \\ \mathrm{w} & \text { at the wall } \\ \theta & \text { in the angular direction }\end{array}$

\section{References}

[1] Westerterp, K. R., van Swaaij, W.P.M., Beenackers, A. A.C.M., Chemical Reactor Design and Operation, 2nd Ed., Wiley, Chichester 1987.

[2] Hiby, J.W., Proc. Symp. Interaction between fluids and particles, London 1962, IChem E, pp. 312-325.

[3] Westerterp, K.R., Wijngaarden, R.J., Dev. Chem. Eng. 14 (1990) pp. $117-128$.

[4] Kirillov, V.A., Matros, Y.S., Sorokin, V.N., Dokl. Akad. SSSR 206 (1972) No. 6, pp. $1409-1414$.

[5] Drahos, J., Cermak, J., Ziolkowska, I., Ziolkowska, D., Chem. Eng. J. 24 (1982) pp. $71-80$.

[6] Price, J., Mech. Eng. Trans. (1968) No. 5, pp. 7- 16.

[7] Vortmeyer, D., Schuster, J., Chem. Eng. Sci. 38, (1983) pp. $1691-1699$.

[8] Vortruba, J., Hlavacek, V., Marek, M., Chem. Eng. Sci. 27 (1972) pp. $1845-1851$

[9] Golay, M. J.E., Savitzky, A., Analyt. Chem. 36 (1964) pp. $1627-1639$.

[10] Michelsen, M.L., Østergaard, K., Chem. Eng. Sci. 25 (1970), pp. 583-592.

[11] Van Gelder, K. B., Westerterp, K. R., Chem. Eng. Technol. 13 (1990) pp. $27-40$. 